\title{
A multi-class classification model for supporting the diagnosis of type II diabetes mellitus
}

\author{
Kuang-Ming Kuo ${ }^{1}$, Paul Talley ${ }^{2}$, YuHsi Kao ${ }^{3}$, Chi-Hsien Huang ${ }^{\text {Corresp. } 4,5}$ \\ ${ }^{1}$ Department of Healthcare Administration, I-Shou University, Kaohsiung City, Taiwan, Republic of China \\ 2 Department of Applied English, I-Shou University, Kaohsiung City, Taiwan, Republic of China \\ 3 Department of Endocrinology, E-Da Hospital, Kaohsiung City, Taiwan, Republic of China \\ 4 Department of Family Medicine, E-Da Hospital, I-Shou University, Kaohsiung City, Taiwan, Republic of China \\ Corresponding Author: Chi-Hsien Huang \\ Email address: evaairgigaa@gmail.com
}

Background. Numerous studies have utilized machine-learning techniques to predict the early onset of type 2 diabetes mellitus. However, fewer studies have been conducted to predict an appropriate diagnosis code for the type 2 diabetes mellitus condition. Further, ensemble techniques such as bagging and boosting have likewise been utilized to an even lesser extent. The present study aims to identify appropriate diagnosis codes for type 2 diabetes mellitus patients by means of building a multi-class prediction model which is both parsimonious and possessing minimum features. In addition, the importance of features for predicting diagnose code is provided.

Methods. This study included 149 patients who have contracted type 2 diabetes mellitus. The sample was collected from a large hospital in Taiwan from November, 2017 to May, 2018. Machine learning algorithms including instance-based, decision trees, deep neural network, and ensemble algorithms were all used to build the predictive models utilized in this study. Average accuracy, area under receiver operating characteristic curve, Mathew correlation coefficient, macro-precision, recall, weighted average of precision and recall, and model process time were subsequently used to assess the performance of the built models. Information gain and gain ratio were used in order to demonstrate feature importance.

Results. The results showed that most algorithms, except for deep neural network, performed well in terms of all performance indices regardless of either the training or testing dataset that were used. Ten features and their importance to determine the diagnosis code of type 2 diabetes mellitus were identified. Our proposed predictive model can be further developed into a clinical diagnosis support system or integrated into existing healthcare information systems. Both methods of application can effectively support physicians whenever they are diagnosing type 2 diabetes mellitus patients in order to foster better patient-care planning. 


\section{A Multi-class Classification Model for Supporting the 2 Diagnosis of Type II Diabetes Mellitus}

3

4 Kuang-Ming Kuo ${ }^{1}$, Paul Talley ${ }^{2}$, YuHsi, Kao ${ }^{3}$, Chi-Hsien Huang 4,5

5

6

$7 \quad{ }^{1}$ Department of Healthcare Administration, I-Shou University, Kaohsiung City, Taiwan

$8 \quad 2$ Department of Applied English, I-Shou University, Kaohsiung City, Taiwan

$9 \quad{ }^{3}$ Department of Endocrinology, E-Da Hospital, Kaohsiung City, Taiwan

$10{ }^{4}$ Department of Family Medicine, E-Da Hospital, I-Shou University, Kaohsiung City, Taiwan

$11{ }^{5}$ Department of Community Healthcare and Geriatrics, Nagoya University Graduate School of

12 Medicine, Nagoya, Japan

14 Corresponding Author:

15 Chi-Hsien Huang 4,5

1665 Tsurumai-cho, Showa-Ku, Nagoya, 466-8550

17 Mobile: +81-70-12641790

18 E-mail: evaairgigaa@gmail.com 


\section{Abstract}

23 Background. Numerous studies have utilized machine-learning techniques to predict the early

24 onset of type 2 diabetes mellitus. However, fewer studies have been conducted to predict an

25 appropriate diagnosis code for the type 2 diabetes mellitus condition. Further, ensemble

26 techniques such as bagging and boosting have likewise been utilized to an even lesser extent.

27 The present study aims to identify appropriate diagnosis codes for type 2 diabetes mellitus

28 patients by means of building a multi-class prediction model which is both parsimonious and

29 possessing minimum features. In addition, the importance of features for predicting diagnose

30 code is provided.

31 Methods. This study included 149 patients who have contracted type 2 diabetes mellitus. The

32 sample was collected from a large hospital in Taiwan from November, 2017 to May, 2018.

33 Machine learning algorithms including instance-based, decision trees, deep neural network, and

34 ensemble algorithms were all used to build the predictive models utilized in this study. Average

35 accuracy, area under receiver operating characteristic curve, Matthew correlation coefficient,

36 macro-precision, recall, weighted average of precision and recall, and model process time were

37 subsequently used to assess the performance of the built models. Information gain and gain ratio

38 were used in order to demonstrate feature importance.

39 Results. The results showed that most algorithms, except for deep neural network, performed

40 well in terms of all performance indices regardless of either the training or testing dataset that

41 were used. Ten features and their importance to determine the diagnosis code of type 2 diabetes

42 mellitus were identified. Our proposed predictive model can be further developed into a clinical

43 diagnosis support system or integrated into existing healthcare information systems. Both

44 methods of application can effectively support physicians whenever they are diagnosing type 2

45 diabetes mellitus patients in order to foster better patient-care planning.

46

Keywords: Diagnosis, machine-learning techniques, predictive models, type 2 diabetes mellitus. 


\section{Introduction}

50 Diabetes mellitus (DM), as defined by the American Diabetes Association (2010), refers to a

51

52

53

54

55

56

57

58

59

60

61

62

63

64

65

66

67

68

69

70

71

72

73

74

75

76

77

78

79

80

81

82

83

84

85

86

87

88 group of metabolic disorders primarily induced by impaired insulin secretion and/or action. Insulin deficiency and increased insulin resistance may lead to an elevated blood glucose level and impaired metabolism of carbohydrates, fat, and protein (American Diabetes Association 2010). DM is one of the most prevalent endocrine disorders, influencing more than 200 million people universally (Kavakiotis et al. 2017). DM has therefore become a global public health challenge, and it is a key health concern worldwide. DM is expected to increase dramatically, and it could potentially be the seventh-leading reason of death in 2030 (World Health Organization 2016). In terms of health-related issues, DM can lead to other serious medical complications such as chronic kidney disease, acute kidney injury, cardiovascular disease, ischemic heart disease, stroke or even to death (World Health Organization 2016). The direct and indirect estimated total cost of diabetes management in the U.S. in 2012 was \$245 billion and increased to $\$ 327$ billion in 2017 (Centers for Diseases Control and Prevention 2017). The burden of DM is rapidly increasing on a global basis and has become a major public health concern. On the other hand, despite the possibly-related complications, DM can be appropriately managed with a comprehensive care plan, such as with a reasonable lifestyle change and significant medication control (American Diabetes Association 2015).

There are two prevalent types of DM, including type 1 diabetes and type 2 diabetes (T2DM), according to the etio-pathology of the disorder (Maniruzzaman et al. 2017). T2DM, accounting for $90 \%$ of DM patients, is the most common form of diabetes (Maniruzzaman et al. 2017). Several risk factors which include smoking, overweight and obesity, physical inactivity, high blood pressure, high cholesterol, and high blood glucose levels were reported to be associated with T2DM (Centers for Diseases Control and Prevention 2017). However, the links between T2DM and some risk factors still remain unclear (Eckel et al. 2011). Currently, the diagnosis of T2DM can be based on elevated Hemoglobin A1c, high fasting or random plasma glucose, and a clinical manifestation of increased urinary frequency (polyuria), thirst (polydipsia), and hunger (polyphagia) (American Diabetes Association 2010). However, it has been estimated that nearly 7.2 million people (23.8\% of diabetes patients) remain undiagnosed in the United States (Centers for Diseases Control and Prevention 2017). Hence, there is a rising need for related research to early identify and to confirm T2DM diagnosis more efficiently and accurately in clinical settings (Kagawa et al. 2017).

Information technologies such as machine-learning techniques have become a vital instrument in determining T2DM diagnosis and affecting management for health care providers and patients (Rigla et al. 2017). Numerous studies have utilized machine-learning techniques to predict the onset of T2DM. While previous DM prediction studies have shown a potential for detecting the onset of T2DM (Alghamdi et al. 2017; Anderson et al. 2015; Esteban et al. 2017; Kagawa et al. 2017; Maniruzzaman et al. 2017; Nilashi et al. 2017; Pei et al. 2019; Talaei-Khoei \& Wilson 2018; Upadhyaya et al. 2017; Wu et al. 2018), no studies, to our knowledge, have been aimed at predicting a suitable diagnosis code for T2DM patients. Further, ensemble machine-learning 
89

90

91

92

93

94

95

96

97

98

99

100

101

102

103

104

105

106

107

108

109

110

111

112

113

114

115

116

117

118

119

120

121

122

123

124

125

126

127

128

techniques such as bagging and boosting approaches are less utilized in these studies (Esteban et al. 2017). Most importantly, less multi-class studies, to our knowledge, have been conducted (Esteban et al. 2017). Therefore, the intended purpose of this study is to leverage routinely available clinical data in order to establish a multi-class predictive model based on bagging and boosting machine-learning techniques useful to identify Asian T2DM patients with a corresponding diagnosis code. The major contribution of our proposed predictive model is its ability to identify a corresponding ICD-10-CM code, not just to identify the onset of T2DM. The correct identification of ICD-10-CM code for T2DM can help physicians and patients form a proper patient-care plan, thus improving the conditions of T2DM patients while reducing the associated heavy financial burden caused by T2DM.

The remainder of this paper is organized as follows: In section 2, we briefly introduce artificial neural networks, decision trees, ensemble models, and support vector machine. In section 3, we present the review of T2DM related studies that used machine-learning techniques. In section 4, we explain the methodology used for data collection, preparation, and analysis in this study. In section 5 , we present the results and in section 6 , we discuss the findings of this study. Finally, in section 7 , we summarize and conclude this study.

\section{Machine learning algorithms}

\section{Artificial neural network}

An artificial neural network (ANN) involves the development of models that enable computers to learn in ways similar to the human brain (Ciaburro \& Venkateswaran 2017; Larrañaga et al. 2019). An ANN is usually organized in layers which comprise a number of interconnected and weighted nodes (or neurons) (Clark 2013; Lantz 2015). To constitute an ANN, at least three layers, including an input layer, a hidden layer, and an output layer, should be included (Lewis 2016).

Fig. 1 shows the relations between input nodes $\left(x_{i}\right)$ and the output node $(y)$. Each of the input nodes is weighted $\left(w_{i}\right)$ based on its importance (Beysolow II 2017). The input nodes are then summed and passed on according to the activation function (Clark 2013; Lantz 2015). An activation is the mechanism by which the artificial neuron handles incoming information and disseminates it all over the network (Lewis, 2016).

$<$ Please insert Fig. 1 here $>$

\section{Decision trees}

Decision trees utilize a tree structure to model the associations found among features and the possible outcomes (Provost \& Fawcett 2013). As Fig. 2 shows, a decision to be considered starts at the root node (Faul 2020), and a decision is made based on the questions of whether the value is higher or lower than a threshold (Brownlee 2017). These decisions then split the data across branches indicating likely outcomes of a decision (Clark 2013). If a final decision can be 
129 reached, the tree is terminated by terminal nodes (Faul 2020). There are many implementations

130 of decision trees, one of the most famous is the C5.0 algorithm, an improvement of C4.5

131 algorithm (Quinlan 1996), and has become a de-facto standard to create decision trees.

132

133

134

135

136

137

138

139

140

141

142

143

144

145

146

147

148

149

150

151

152

153

154

155

156

157

158

159

160

161

162

163

164

165

166

167

168

$<$ Please insert Fig. 2 here $>$

\section{Ensemble model (Bagging and boosting)}

The technique of merging and managing the predictions of multiple models is known as an ensemble approach (Lantz 2015). More specifically, ensemble methods are hinged on the notion that by merging multiple weaker learners, a stronger learner is generated (Clark, 2013). Bagging and boosting are widespread accepted ensemble methods currently.

\section{Bagging}

One of the ensemble approaches to receive widely acknowledgement adopted a technique named bootstrap aggregating or bagging, to generate a number of datasets for training by bootstrap sampling from the primitive training dataset (Lantz 2015). These data are then utilized to create a set of models with each incorporating only one classifier. Averaging (for numeric prediction) or voting (for classification) are used to determine the model's terminal predictions (Beysolow II 2017; Clark 2013). Among many bagging classifiers, random forest, a combination of several decision trees (Beysolow II 2017), merges the basic rules of bagging with random feature selection to increase additional variety to the building of the models. After the ensembles of trees is created, the model utilizes a vote to merge the tree's predictions (Beysolow II 2017).

\section{Boosting}

Another ensemble-based method is known as boosting since it is a method of boosting weak learners to become strong learners (Beysolow II 2017; Brownlee 2017; Faul 2020). In boosting, each new tree is a fit on an adjusted version of the primitive dataset. Different from bagging, boosting resampled datasets are constructed to generate complementary learners, and boosting gives each learner's vote a weight based on its past performance (Lantz 2015).

Among the many boosting classifiers, eXtreme gradient boosting (Chen \& Guestrin 2016) is one of the most popular applications of gradient boosting concept. This classifier is basically designed to enhance the performance and speed of a machine learning model. What makes eXtreme gradient boosting peculiar is that it utilizes a more regularized model formalization to regulate over-fitting, which thus gives it better performance (Lantz 2015).

\section{Support vector machine}

A support vector machine (SVM), an instance-based algorithm, tries to maximize the margin between two classes by using kernel function (Marsland 2015). In other words, SVM creates a boundary called a hyperplane (Beysolow II 2017) and tries to search for the maximum margin hyperplane (Brownlee, 2017), which breaks the space to create the best homogenous partitions 
169

170

171

172

173

174

175

176

177

178

179

180

181

182

183

184

185

186

187

188

189

190

191

192

193

194

195

196

197

198

199

200

201

202

203

204

205

206

207

208

on two different classes (see Fig. 3). The support vectors are the points from each class that are the nearest to the maximum margin hyperplane, which is a key feature of SVMs (Lantz 2015). SVMs can be utilized along with almost any type of learning task, including numeric prediction and classification (Kuhn \& Johnson 2013).

$<$ Please insert Fig. 3 here $>$

\section{Related work}

Thus far, a large number of studies have attempted to predict the onset of T2DM based on differing machine-learning algorithms. The study by Kavakiotis et al. (2017) provides an excellent review on machine-learning and data-mining methods in prior diabetes research. To prevent reiteration of what Kavakiotis et al. (2017) have found, we reviewed T2DM studies made during 2015-2019 that utilized machine-learning techniques, as follows :1) adopted machine-learning algorithms, 2) the features used to predict T2DM, 3) the sample locations experienced, and 4) classification type:

\section{Adopted machine learning algorithms}

Several types of machine-learning algorithms, including instance-based (Esteban et al. 2017; Kagawa et al. 2017; Nilashi et al. 2017; Pei et al. 2019; Talaei-Khoei \& Wilson 2018), decision trees (Alghamdi et al. 2017; Esteban et al. 2017; Pei et al. 2019; Talaei-Khoei \& Wilson 2018), artificial neural network (Esteban et al. 2017; Nilashi et al. 2017; Talaei-Khoei \& Wilson 2018), ensemble (Alghamdi et al. 2017; Esteban et al. 2017; Pei et al. 2019), Bayesian (Alghamdi et al. 2017; Anderson et al. 2015; Esteban et al. 2017; Maniruzzaman et al. 2017; Pei et al. 2019), statistical model (Alghamdi et al. 2017; Esteban et al. 2017; Maniruzzaman et al. 2017; TalaeiKhoei \& Wilson 2018; Wu et al. 2018), and others (see Table 1), have been adopted to predict T2DM-related issues. However, these studies revealed different results in predicting the onset of T2DM even with the same machine-learning algorithm. For example, Pei et al. (2019) and Alghamdi et al. (2017) both adopted J48 as one of their algorithms for predicting the onset of T2DM, only Pei et al. (2019) found that J48 had the best performance. The performance of support vector machine also differs among opposing studies (Esteban et al. 2017; Kagawa et al. 2017; Nilashi et al. 2017; Pei et al. 2019; Talaei-Khoei \& Wilson 2018). Further, not all inclusionary studies adopted the same algorithms, making it difficult to accurately compare the performance of differing algorithms. Finally, artificial neural network were adopted by three studies (Esteban et al. 2017; Nilashi et al. 2017; Talaei-Khoei \& Wilson 2018) and outperformed other algorithms in these respective studies. Further, deep-learning techniques were not applied in these T2DM-related studies.

$<$ Please Insert Table 1 here $>$

\section{Features used to predict T2DM}


209 Regarding features selected to predict the onset of T2DM, they can be roughly classified into 210 five major categories (see Table 2): 1) demographic data; 2) laboratory test results; 3) vital signs;

211 4) life style; and, 5) history. Demographic data such as age, gender, Body Mass Index (BMI)

212 were often adopted features used for predicting the onset of T2DM (Alghamdi et al. 2017;

213 Anderson et al. 2015; Pei et al. 2019; Talaei-Khoei \& Wilson 2018). Laboratory tests such as fast

214 plasma glucose, Hemoglobin A1c, high-density lipoprotein cholesterol were commonly seen

215 features used by the regarded T2DM studies (Anderson et al. 2015; Maniruzzaman et al. 2017;

216 Talaei-Khoei \& Wilson 2018; Upadhyaya et al. 2017; Wu et al. 2018). Vital signs such as

217 diastolic blood pressure and systolic blood pressure were also used by those reviewed studies

218 (Alghamdi et al. 2017; Nilashi et al. 2017; Talaei-Khoei \& Wilson 2018; Wu et al. 2018). Life-

219 style features such as physical activity, work stress, salty-food preference (Pei et al. 2019),

220 shortness of breath, frequent urination at night, excessive thirst (Maniruzzaman et al. 2017;

221 Nilashi et al. 2017; Talaei-Khoei \& Wilson 2018), and sedentary lifestyle (Alghamdi et al. 2017)

222 were also included. Finally, history features such as family history of diabetes (Maniruzzaman et

223 al. 2017; Nilashi et al. 2017; Pei et al. 2019; Talaei-Khoei \& Wilson 2018) or prescriptions of

224 diabetes-related medication (Esteban et al. 2017; Kagawa et al. 2017; Upadhyaya et al. 2017)

225 were included. In practice, it is not easy to collect all the features utilized in the above-discussed

226 studies since some features require extra efforts to acquire. It will therefore be more practical and

227 efficient to collect required data, for prediction of the onset of T2DM, from Electronic Medical

228 Records since most hospitals have extensively used Electronic Medical Records to assist patient

229 care.

230

231

232

233

234

235

236

237

238

239

240

241

242

243

244

245

246

\section{Sample locations experienced}

As shown in Table 3, we can find that most samples were taken in the United States (Alghamdi et al. 2017; Anderson et al. 2015; Maniruzzaman et al. 2017; Nilashi et al. 2017; Upadhyaya et al. 2017; Wu et al. 2018), while fewer study samples were drawn from Asian countries (Kagawa et al. 2017; Pei et al. 2019). The evidence reviewed here clearly highlights the present need that more diversified samples should be included and analyzed aiming to better clarify their relations with the onset of T2DM.

$<$ Please Insert Table 3 here $>$

247 
248 According to a review of recent T2DM studies that utilized machine-learning techniques, several 249 points should be duly noted. First, how different algorithms perform in predicting the onset of 250 T2DM is still unclear and incomparable since each of the studies adopted differing algorithms.

251 Second, deep learning and ensemble approaches are utilized to a lesser extent than in those

252 reviewed studies. Third, no clear results demonstrate which features should be used to predict the 253 onset of T2DM. Fourth, T2DM patients from Asian countries were under-represented in studies 254 using machine-learning techniques than from outside the U.S. As a result, these findings are not 255 comparable or contrastive in achieving a better understanding of the various aspects regarding 256 T2DM. Fifth, owing to an increasing number of disease sub-categories, it is mandatory to conduct a multi-class study to facilitate and then to confirm final diagnosis. For example, most

258

259

260

261

\section{Materials \& Methods}

263

264

265

266

267

268

269

270

271

272

273

274

275

276

277

278

279

280

281

282

283

284

285

286

287 ever investigated.

\section{Data}

reviewed studies demonstrated the potential for predicting the onset of T2DM, whereas the onset of T2DM complications, including as retinopathy, neuropathy, and nephropathy, were rarely if

Diagnosing T2DM depends primarily on laboratory test results (American Diabetes Association 2010), we therefore required a collection of those data from T2DM patients. A plausible T2DM patient list was first obtained, containing patients who had visited an endocrinologist (one of our authors) between November, 2017 and May, 2018 at a large hospital in southern Taiwan. The Institutional Review Board of E-Da Hospital approved our study protocol and waived informed consent regarding this study (EMRP-107-048). In consideration of the features to be included, we elected to adopt ten common features based on our review of prior studies related to DM prediction models (Anderson et al. 2015; Pei et al. 2019; Talaei-Khoei \& Wilson 2018; Wu et al. 2018). These readily available features can be drawn directly or indirectly from the content of Electronic Medical Records. By doing so, the predictive model we proposed can be adopted by most hospitals since these selected features are already stored in existing databases.

The ten health-related features can be primarily classified into two categories: demographic data and laboratory test results. Demographic data included age, gender, smoking status, and BMI which were reported to be associated with the onset of T2DM (Yuan et al. 2018). On the other hand, laboratory data are comprised of total cholesterol, triglyceride, glucose (AC), Hemoglobin A1c, high-density lipoprotein cholesterol, and low-density lipoprotein cholesterol which were indicators of impaired metabolic function pre-disposing DM (Guasch-Ferré et al. 2016). Eligibility criteria for the study were that a patient must (1) be diagnosed through an international classification of diseases, tenth revision, clinical modification (ICD-10-CM) starting with E11, and (2) no missing data in total cholesterol, triglyceride, glucose (AC), Hemoglobin A1c, high-density lipoprotein cholesterol, and low-density lipoprotein cholesterol was evident. Initially, a total of 10,527 plausible T2DM patient information were obtained and duplicated patient listings were first removed. Patients with missing laboratory test results were then removed. Since there may be many ICD-10-CM codes utilized for diagnosing T2DM, we

Peer) reviewing PDF | (2020:05:48812:2:0:NEW 11 Aug 2020) 
288 limited our predicted classes to the first five digits of the ICD-10-CM code; and as such, these 289 five-digit codes must be among the top ICD-10-CM codes appearing in our collected data. 290 Finally, 149 eligible records, including E1121 (T2DM with diabetic nephropathy, n=45), E1143 291 (T2DM with diabetic autonomic [poly]neuropathy, $\mathrm{n}=88$ ), and E1165 (T2DM with 292 hyperglycemia, $\mathrm{n}=16$ ), without missing values were collected.

293 Our inclusion of these ten features primarily differs from prior T2DM-related studies in that we 294 only included demographic data and laboratory test results, while prior T2DM studies included a 295 wider variety of data. In words, we aimed to build a parsimonious predictive model possessing 296 minimum features. Table 4 shows the detailed operational definition of features used in our 297 study.

298

299

300

301

302

303

304

305

306

307

308

309

310

311

312

313

314

315

\section{Experimental setup}

$<$ Please Insert Table 4 here $>$

To predict a diagnosis code for the T2DM patient, we adopted R 4.0.0 software (R Core Team 2020) for purposes of data analysis. Since our data is non-linear, machine-learning techniques are well-suited for predicting the ICD-10-CM code of T2DM. Based on the methodological gaps found in our review of T2DM related studies, we decided to choose five machine-learning algorithms including instance-based (Support vector machine), decision trees (C5.0), deep neural network, and ensemble (Random forest and eXtreme gradient boosting) as primary learners in our study.

We used the mlr 2.17.1 package (Bischl et al. 2016) to automatically tune the optimal model parameters for these four learners aiming to obtain a better level of predictive performance. The $\mathrm{R}$ packages used for machine-learning algorithms and their respective optimal model parameters are shown in Table 5. Further, since our predicted class is imbalanced, we utilized a synthetic minority over-sampling technique provided by UBL package (Branco et al. 2016) in order to improve the model performance.

316

317

318

319

320

321

322

323

324

325

326

$<$ Please Insert Table 5 here $>$

We adopted: 1) 10-fold cross-validation; 2) leave-one-subject-out; and 3) holdout approaches to assess the performance of the five learners. The 10-fold cross-validation approach randomly splits the dataset into 10 subsets with roughly similar sizes, among which nine subsets are used for constructing the model and the remaining one subset is utilized for testing the model (Provost $\&$ Fawcett 2013). Leave-one-subject-out cross-validation is a special case of k-fold crossvalidation since $\mathrm{k}$ is the number of samples while holdout simply splits data into training samples for building the predictive model and testing samples for estimating model performance (Kuhn \& Johnson 2013).

\section{Performance metrics}


327

328

329

330

331

332

333

334

335

336

337

338

339

340

341

342

343

344

345

346

347

348

349

350

351

352

353

354

355

356

357

358

359

360

361

362

363

364

365

366

To better evaluate the performance of a multi-class setting, we employed average accuracy, area under receiver operating characteristic (AUC), Matthew correlation coefficient (MCC), and the macro-averaging of precision, recall, and $\mathrm{F} 1$ score (weighted average of precision and recall) according to the suggestions taken from the literature (Sokolova \& Lapalme 2009). These metrics were measured based on a confusion matrix (see Table 6).

$<$ Please Insert Table 6 here $>$

The average accuracy, Matthew correlation coefficient, micro- and macro-averaging precision, recall, and F1 score were then acquired using the formulae located in Table 7.

$<$ Please Insert Table 7 here $>$

Regarding the interpretation of these metrics, the average accuracy, AUC, MCC, macroaveraging and micro-averaging precision, recall, and F1 score value between 0 and 1, with values approaching 1 , imply better performance.

\section{Results}

\section{Data profiles}

Table 8 demonstrates the descriptive statistics for T2DM patients. Among these figures, the proportion of the male sample is higher than that of female, aged 21-91 years, and most samples did not smoke, or had quit smoking, at the time of survey administration. Furthermore, the average BMI of samples belonging to the 'obesity' level, and the average levels of glucose (AC) and Hemoglobin A1c are higher than the normal values. On average, other laboratory test results fall inside the normal range.

$<$ Please Insert Table 8 here $>$

\section{Model performance}

Under 10-fold cross-validation, the performance of support vector machine ranked the highest in accuracy, AUC, MCC, macro-averaging F1 score, macro-averaging precision, and macroaveraging recall metrics with training samples (see Table 9). This was followed by random forest, C5.0, deep neural network, and eXtreme gradient boosting. Further, the process time for training the support vector machine was also the shortest compared to the remaining algorithms. When comparing the performance of the five trained models in the test samples, support vector machine, C5.0, and random forest perfectly achieved 1 in accuracy, AUC, MCC, macroaveraging F1 score, macro-averaging precision, and macro-averaging recall metrics (see Table 9 and Fig. 4). eXtreme gradient boosting learner also achieved higher than 0.9 in all metrics. Deep neural network however performed poorer than the other four learners in all metrics. We then compared the model performance by use of the Stuart-Maxwell test which is better suited for 
367

368

369

370

371

372

373

374

375

376

377

378

379

380

381

382

383

384

385

386

387

388

389

390

391

392

393

394

395

396

397

398

399

400

401

402

403

404

405

406

multi-class classification models than McNemar test (Maxwell 1970; McNemar 1947; Stuart 1955). Since support vector machine, C5.0, and random forest perfectly predicted ICD-10-CM codes used for T2DM, we only statistically compared the performance of deep neural network and eXtreme gradient boosting learners. The Stuart-Maxwell tests demonstrated significant results for both deep neural network $(p<0.001)$ and eXtreme gradient boosting $(p=0.002)$, thus indicating significant difference disagreement between these two algorithms and the observed data.

$<$ Please Insert Table 9 here $>$

$<$ Please Insert Fig. 4 here $>$

Under leave-one-subject-out cross-validation, both support vector machine and random forest performed better than the remaining classifiers, with training samples, in terms of all metrics, including process time (see Table 10). As for the model performance of testing samples, support vector machine, C5.0, and random forest perfectly achieved 1 in accuracy, AUC, MCC, macroaveraging F1 score, macro-averaging precision, and macro-averaging recall metrics (see Table 10 and Fig. 5). Deep neural network and eXtreme gradient boosting still did not perform as well as the remaining classifiers. Stuart-Maxwell tests were then conducted for deep neural network and eXtreme gradient boosting. And, the results revealed that deep neural network still showed significant difference with the observed data $(p<0.001)$ while eXtreme gradient boosting showed insignificant difference with the observed data $(p=0.06)$.

$<$ Please Insert Table 10 here $>$

$<$ Please Insert Fig. 5 here $>$

Under hold-out cross-validation, support vector machine still performed better than the remaining classifiers, with training samples, in terms of all metrics, including process time (see Table 11 and Fig. 6). Deep neural network and eXtreme gradient boosting still did not perform as well as the remaining classifiers. The Stuart-Maxwell tests demonstrated significant results for both deep neural network $(p<0.001)$ and eXtreme gradient boosting $(p=0.018)$, indicating significant difference disagreement between these two algorithms and the observed data.

$<$ Please Insert Table 11 here $>$

$<$ Please Insert Fig. 6 here $>$

\section{Feature importance}

In addition to making a comparison of the performance for the four prediction models, we also ranked the feature importance based on information gain and gain ratio (see Fig. 7). Information gain can be biased if features have a large number of possible outcomes, which may be corrected by gain ratio criteria (Kuhn \& Johnson 2013). From the perspective of information gain, or 
407 precisely how much a feature improves entropy (a measure of disorder), Hemoglobin A1c, age, 408 triglyceride, low-density lipoprotein cholesterol, high-density lipoprotein cholesterol, and total 409 cholesterol ranked as the top six most important features for predicting ICD-10-CM code. After 410 correcting for possible bias, High-density lipoprotein cholesterol, Hemoglobin A1c, age, low411 density lipoprotein cholesterol, triglyceride, and total cholesterol ranked as the top six important 412 features. The greatest difference in the rankings, based upon information gain and gain ratio, is 413 high-density lipoprotein cholesterol, ranked 5th by information gain, but ranked 1st by gain ratio. 414 Further, BMI and glucose did not contribute anything to the class prediction of ICD-10-CM code 415 for T2DM.

416

417

$<$ Please Insert Fig. 7 here $>$

418

419

420

421

422

423

424

425

426

427

\section{Discussion}

As mentioned at the beginning of our study, T2DM should be considered as a catastrophic threat to public health that is accompanied by huge financial and personal costs following the onset of T2DM. Therefore, obtaining the means of how to correctly diagnose T2DM patients in order to foster appropriate medical care for T2DM patients is inevitable and of great importance to the health-care profession. This study aimed to build an appropriate model for predicting ICD-10CM code by utilizing bagging and boosting ensemble techniques for Asian T2DM patients. Our proposed model, based on support vector machine, performed well in terms of average accuracy, AUC, MCC, macro-averaging F1 score, macro-averaging precision, and macro-averaging recall.

428

429

430

431

432

433

434

435

436

437

438

439

440

441

442

443

444

445

446 Based on information gain and gain ratio, our study also distinguished and ranked the top eight variables, including Hemoglobin A1c, age, triglyceride, low-density lipoprotein cholesterol, high-density lipoprotein cholesterol, and total Cholesterol, along with the habit of smoking, to predict ICD-10-CM codes for T2DM patients.

Although the performance metrics are not entirely consistent among T2DM-related studies that used machine-learning technique, it is still worthwhile to make a comparison between the current study and those studies with available performance metrics (see Table 12). Support vector machine was the best classifier in our study with accuracy, AUC, MCC, macro-averaging F1 score, macro-averaging precision, and macro-averaging recall metrics all equal to 1 . Prior studies utilized support vector machine also performed well but only in some metrics. For example, the study of Pei et al. (2019) achieved 0.908 accuracy rate for diabetes classification with noninvasive and easily gathered features. Talaei-Khoei \& Wilson (2018) used machine-learning techniques to identify people at risk of developing T2DM and found the MCC metric of support vector machine was 0.922 . Kagawa et al. (2017) combined expert knowledge and machinelearning approaches to determine whether a patient has T2DM. Among the five classifiers adopted, support vector machine achieved 0.909 in recall metric.

Regarding studies that adopted neural network classifier, the study of Nilashi et al. (2017) achieved 0.923 accuracy rate while the study of Esteban et al. (2017) achieved 0.93 and 0.96 for precision and recall metrics, respectively. Finally, random forest also perfectly predicted ICD- 
447 10-CM code in our study for accuracy, AUC, MCC, macro-averaging F1 score, macro-averaging 448 precision, and macro-averaging recall metrics. Alghamdi et al. (2017) adopted random forest to

449

450

451

452

453

454

455

456

457

458

459

460

461

462

463

464

465

466

467

468

469

470

471

472

473

474

475

476

477

478

479

480

481

482

483

484

485

486

\section{$<$ Please Insert Table 12 here $>$}

Several interesting points can be derived from our findings as a whole. First, as suggested by prior literature (Lantz 2015), our proposed predictive model implementing ensemble method (i.e., random forest and eXtreme gradient boosting) has performed, despite not being the best, satisfactorily with average accuracy, AUC, MCC, macro-averaging F1 score, macro-averaging precision, and macro-averaging recall being higher than 0.97 for all metrics among three resampling strategies. Future research may prove to implement these techniques that will lead to improved model predictive power.

Second, by using Asian samples, the findings determined in our study can be further compared with prior similar studies, and attention can be placed on the differences. For example, the link between obesity and T2DM remains uncertain (Eckel et al. 2011), BMI ranked the ninth important feature for predicting T2DM diagnosis in terms of both information gain and gain ratio. Future research can further explore why and how this difference comes to exist between eastern and western population samples.

Third, differing from most prior studies, our proposed models aimed to predict a multi-class classification task, which may provide more accurate predictions over and above binary classification tasking (Zhou et al. 2016) since there may be numerous features that specifically identify a certain category. It is therefore of practical significance to apply a multi-class classification approach useful to predict ICD-10-CM code for T2DM patients.

Finally, our predictive model can be further developed into a clinical diagnosis support system, or even better when integrated into existing healthcare information systems aiming to support physicians, when diagnosing T2DM patients. By means of such a support system/function, physicians can better diagnose and foster medical care plans for T2DM patients to follow. The ability to predict disease sub-categories may assist and further remind physicians to early detect and manage possible complications in the earliest stages of disease onset.

One of the most important limitations found in our study is that we utilized only three ICD-10$\mathrm{CM}$ codes pertinent to T2DM for predictive purposes. There are in fact many ICD-10-CM codes available for T2DM diagnosis and care; so, it is possible for future research to increase the 
487

488

489

490

491

492

493

494

495

496

497

498

499

500

501

502

503

504

505

506

507

508

509

510

511

512

513

514

515

516

517

518

519

520

521

522

523

524

525

526

527

528

529

number of ICD-10-CM codes in the predicted class in order to broaden diagnostic applications. In order to ensure as complete a data set as possible in building our model, we were required to remove those samples with missing data which resulted in only useable 149 samples extant. Future studies may choose to increase the sample size in order to enhance external the generalizability of the findings.

\section{Conclusions}

Our study adopted machine-learning techniques using ten features adapted from Electronic Medical Records for identifying diagnosis code for T2DM patients. By adopting 10-fold, leaveone-subject-out, and holdout resampling strategy, support vector machine and random forest showed the best classification metrics in identifying an ICD-10-CM code for the test samples. These results demonstrated that our established model successfully achieved predictive and wholly appropriate ICD-10-CM code for T2DM patients to use. The implementation of our established predictive model in conjunction with using machine-learning algorithms along with data from Electronic Medical Records enables an in-depth exploration toward supporting diagnosis of T2DM patients. This approach may be easily applied within healthcare facilities which have implemented complete electronic medical record-keeping.

\section{Acknowledgements}

We want to show gratitude to Su-Ya Pan and Yao-Kun Cheng for carrying out the data application and collection used in this study.

\section{References}

Alghamdi M, Al-Mallah M, Keteyian S, Brawner C, Ehrman J, and Sakr S. 2017. Predicting diabetes mellitus using SMOTE and ensemble machine learning approach: The Henry Ford ExercIse Testing (FIT) project. PloS One 12:e0179805. 10.1371/journal.pone.0179805

American Diabetes Association. 2010. Diagnosis and Classification of Diabetes Mellitus. Diabetes Care 33:S62-S69. 10.2337/dc10-S062

American Diabetes Association. 2015. Standards of medical care in diabetes-2015 abridged for primary care providers. Clinical Diabetes 33:97-111.

Anderson JP, Parikh JR, Shenfeld DK, Ivanov V, Marks C, Church BW, Laramie JM, Mardekian J, Piper BA, Willke RJ, and Rublee DA. 2015. Reverse Engineering and Evaluation of Prediction Models for Progression to Type 2 Diabetes: An Application of Machine Learning Using Electronic Health Records. Journal of Diabetes Science and Technology 10:6-18. $10.1177 / 1932296815620200$

Beysolow II T. 2017. Introduction to Deep Learning Using R: A Step-by-Step Guide to Learning and Implementing Deep Learning Models Using R. San Francisco, California: Apress.

Bischl B, Lang M, Kotthoff L, Schiffner J, Richter J, Studerus E, Casalicchio G, and Jones ZM. 2016. mlr: Machine Learning in R. The Journal of Machine Learning Research 17:1-5.

Branco P, Ribeiro RP, and Torgo L. 2016. UBL: An r Package for Utility-based Learning. Cornell university.

Brownlee J. 2017. Master Machine Learning Algorithms: Discover How They Work and Implement Them From Scratch. 
530

531

532

533

534

535

536

537

538

539

540

541

542

543

544

545

546

547

548

549

550

551

552

553

554

555

556

557

558

559

560

561

562

563

564

565

566

567

568

569

570

571

572

573

574

575

576

577

578

579

580

Centers for Diseases Control and Prevention. 2017. National Diabetes Statistics Report. Available at https://www.cdc.gov/diabetes/data/statistics/statistics-report.html (accessed August 25 2019).

Chen T, and Guestrin C. 2016. XGBoost: A Scalable Tree Boosting System. Proceedings of the 22nd ACM SIGKDD International Conference on Knowledge Discovery and Data Mining. San Francisco, California, USA: Association for Computing Machinery. p 785-794.

Ciaburro G, and Venkateswaran B. 2017. Neural Networks with R: Smart models using CNN, RNN, deep learning, and artificial intelligence principles. Birminghan, UK: Packt Publishing Ltd.

Clark M. 2013. An Introduction to machine learning with Applications in R: Center for Social Research, University of Notre Dame.

Eckel RH, Kahn SE, Ferrannini E, Goldfine AB, Nathan DM, Schwartz MW, Smith RJ, and Smith SR. 2011. Obesity and type 2 diabetes: what can be unified and what needs to be individualized? The Journal of clinical endocrinology and metabolism 96:1654-1663. 10.1210/jc.2011-0585

Esteban S, Rodríguez Tablado M, Peper FE, Mahumud YS, Ricci RI, Kopitowski KS, and Terrasa SA. 2017. Development and validation of various phenotyping algorithms for Diabetes Mellitus using data from electronic health records. Computer Methods and Programs in Biomedicine 152:53-70. 10.1016/j.cmpb.2017.09.009

Faul AC. 2020. A Concise Introduction to Machine Learning. Boca Raton, FL: CRC Press.

Guasch-Ferré M, Hruby A, Toledo E, Clish CB, Martínez-González MA, Salas-Salvadó J, and Hu FB. 2016. Metabolomics in Prediabetes and Diabetes: A Systematic Review and Meta-analysis. Diabetes Care 39:833. 10.2337/dc15-2251

Kagawa R, Kawazoe Y, Ida Y, Shinohara E, Tanaka K, Imai T, and Ohe K. 2017. Development of Type 2 Diabetes Mellitus Phenotyping Framework Using Expert Knowledge and Machine Learning Approach. Journal of Diabetes Science and Technology 11:791-799. 10.1177/1932296816681584

Kavakiotis I, Tsave O, Salifoglou A, Maglaveras N, Vlahavas I, and Chouvarda I. 2017. Machine Learning and Data Mining Methods in Diabetes Research. Computational and Structural Biotechnology Journal 15:104-116. 10.1016/j.csbj.2016.12.005

Kuhn M, and Johnson K. 2013. Applied Predictive Modeling. New York: Springer.

Lantz B. 2015. Machine learning with R. Birmingham, UK: Packt Publishing Ltd.

Larrañaga P, Atienza D, Diaz-Rozo J, Ogbechie A, Puerto-Santana CE, and Bielza C. 2019. Industrial applications of machine learning. Boca Raton, FL: CRC Press.

Lewis ND. 2016. Deep Learning made Easy with R: A Gentle Introduction for Data Science. Scotts Valley, CA.: CreateSpace Independent Publishing Platform.

Maniruzzaman M, Kumar N, Menhazul Abedin M, Shaykhul Islam M, Suri HS, El-Baz AS, and Suri JS. 2017. Comparative approaches for classification of diabetes mellitus data: Machine learning paradigm. Computer Methods and Programs in Biomedicine 152:23-34.

10.1016/j.cmpb.2017.09.004

Marsland S. 2015. Machine Learning: An Algorithm Perspective. Boca Raton, FL: CRC Press.

Maxwell AE. 1970. Comparing the Classification of Subjects by Two Independent Judges. British Journal of Psychiatry 116:651-655. 10.1192/bjp.116.535.651

McNemar Q. 1947. Note on the sampling error of the difference between correlated proportions or percentages. Psychometrika 12:153-157. 10.1007/BF02295996

Nilashi M, Ibrahim O, Dalvi M, Ahmadi H, and Shahmoradi L. 2017. Accuracy Improvement for Diabetes Disease Classification: A Case on a Public Medical Dataset. Fuzzy Information and Engineering 9:345-357. 10.1016/j.fiae.2017.09.006

Pei D, Gong Y, Kang H, Zhang C, and Guo Q. 2019. Accurate and rapid screening model for potential diabetes mellitus. BMC medical informatics and decision making 19:41. 10.1186/s12911-0190790-3

Provost F, and Fawcett T. 2013. Data Science for Business: What you need to know about data mining and data-analytic thinking. Sebastopol, CA: O'Reilly Media, Inc.

Quinlan JR. 1996. Improved Use of Continuous Attributes in C4.5. Journal of Artificial Intelligence Research 4:77-90.

Peer] reviewing PDF | (2020:05:48812:2:0:NEW 11 Aug 2020) 
581

582

583

584

585

586

587

588

589

590

591

592

593

594

595

596

597

598

599

600

601

602

603

604

605

606

607

R Core Team. 2020. R: A language and environment for statistical computing. . Vienna, Austria: R Foundation for Statistical Computing, .

Rigla M, García-Sáez G, Pons B, and Hernando ME. 2017. Artificial Intelligence Methodologies and Their Application to Diabetes. Journal of Diabetes Science and Technology 12:303-310. $10.1177 / 1932296817710475$

Sokolova M, and Lapalme G. 2009. A systematic analysis of performance measures for classification tasks. Information Processing \& Management 45:427-437. 10.1016/j.ipm.2009.03.002

Stuart A. 1955. A Test For Homogeneity Of The Marginal Distributions In A Two-Way Classification. Biometrika 42:412-416. 10.1093/biomet/42.3-4.412

Talaei-Khoei A, and Wilson JM. 2018. Identifying people at risk of developing type 2 diabetes: A comparison of predictive analytics techniques and predictor variables. International Journal of Medical Informatics 119:22-38. 10.1016/j.ijmedinf.2018.08.008

Upadhyaya SG, Murphree DH, Ngufor CG, Knight AM, Cronk DJ, Cima RR, Curry TB, Pathak J, Carter RE, and Kor DJ. 2017. Automated Diabetes Case Identification Using Electronic Health Record Data at a Tertiary Care Facility. Mayo Clinic Proceedings: Innovations, Quality \& Outcomes 1:100-110. 10.1016/j.mayocpiqo.2017.04.005

World Health Organization. 2016. Global Report on Diabetes. Geneva, Switzerland: World Health Organization, Available at https://www.who.int/diabetes/global-report/en/ (accessed 5 June 2020).

Wu H, Yang S, Huang Z, He J, and Wang X. 2018. Type 2 diabetes mellitus prediction model based on data mining. Informatics in Medicine Unlocked 10:100-107. 10.1016/j.imu.2017.12.006

Yuan S, Xue H-1, Yu H-j, Huang Y, Tang B-w, Yang X-h, Li Q-x, and He Q-q. 2018. Cigarette smoking as a risk factor for type 2 diabetes in women compared with men: a systematic review and metaanalysis of prospective cohort studies. Journal of Public Health 41:e169-e176. 10.1093/pubmed/fdy106

Zhou L, Tam KP, and Fujita H. 2016. Predicting the listing status of Chinese listed companies with multiclass classification models. Information Sciences 328:222-236. 10.1016/j.ins.2015.08.036

Peer] reviewing PDF | (2020:05:48812:2:0:NEW 11 Aug 2020) 
Figure 1

Fig. 1 Artificial neural network architecture

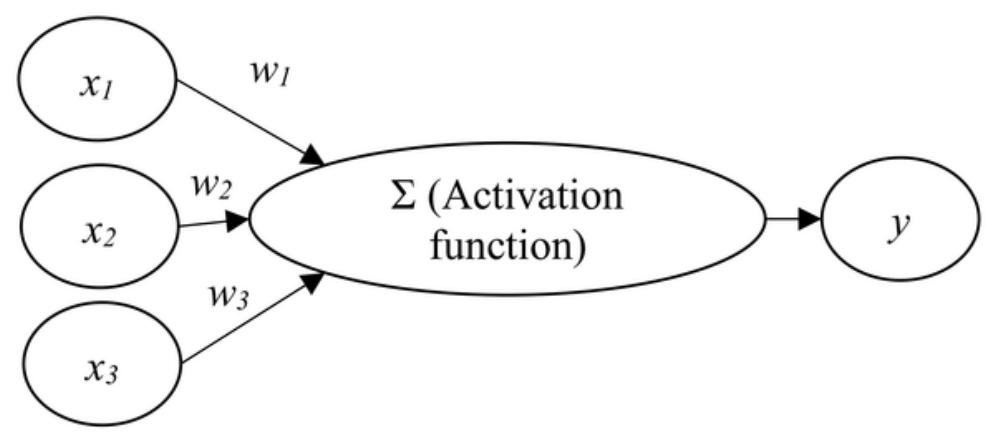


Figure 2

Fig. 2 Decision trees

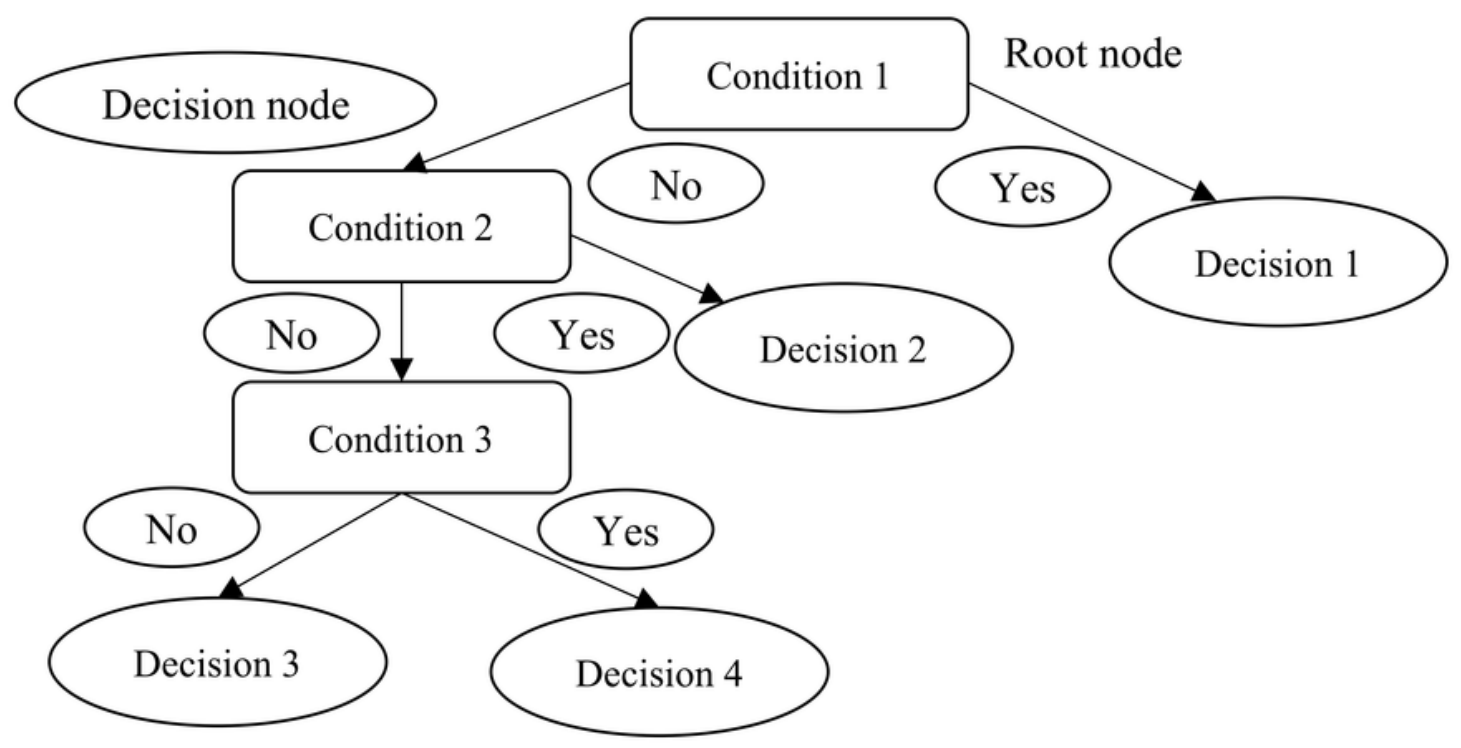


Figure 3

Fig. 3 Margin hyperplane, support vector, and convex hull

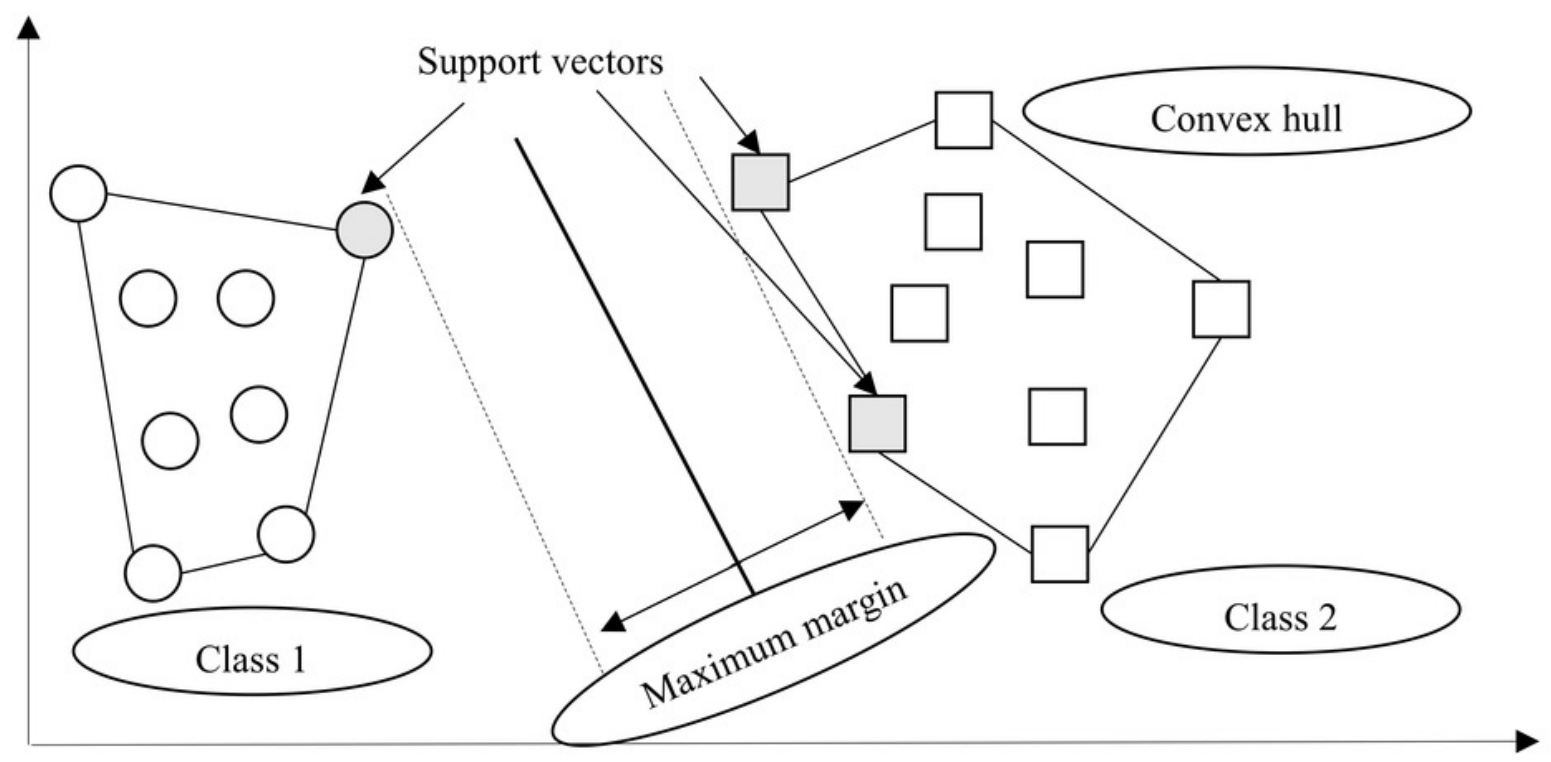


Figure 4

Fig. 4 Model performance of test dataset - 10-fold cross-validation

Note: AUC: area under receiver operating characteristic curve, MCC: Matthew correlation coefficient.

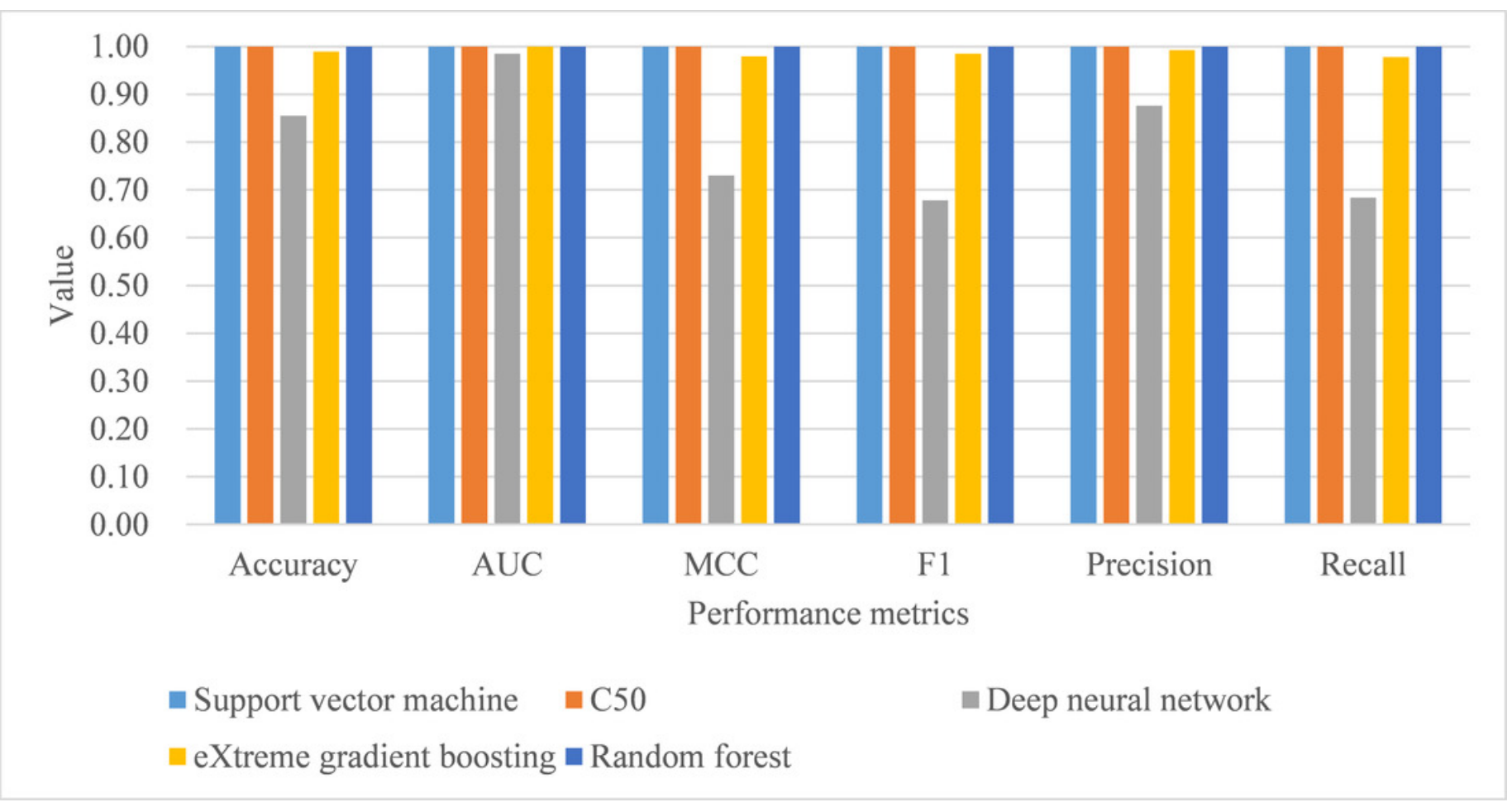


Figure 5

Fig. 5 Model performance of test dataset - Leave-one-subject-out cross-validation

Note: AUC: area under receiver operating characteristic curve, MCC: Matthew correlation coefficient.

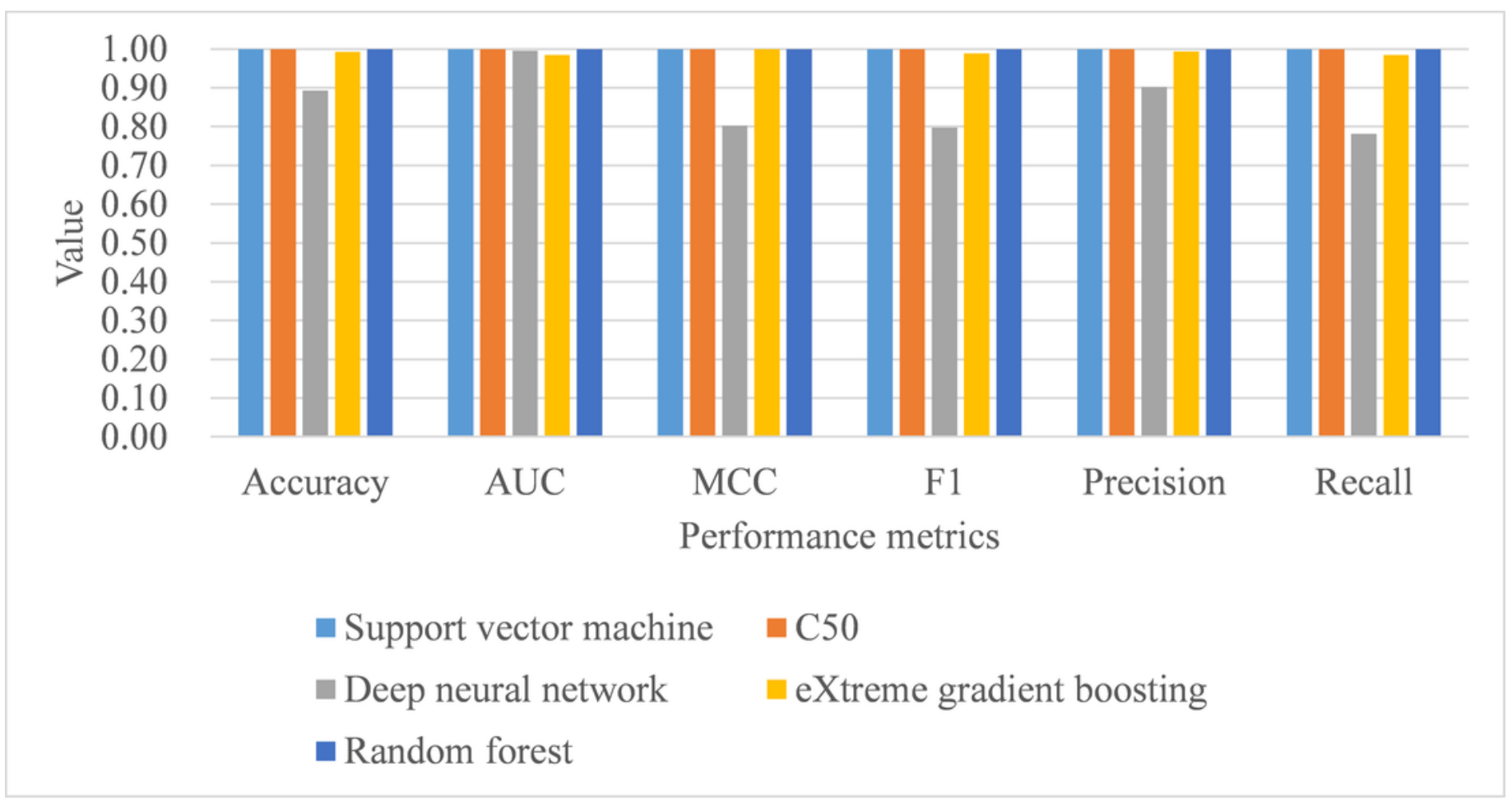




\section{Figure 6}

Fig. 6 Model performance of test dataset - Holdout cross-validation

Note: AUC: area under receiver operating characteristic curve, MCC: Matthew correlation coefficient.

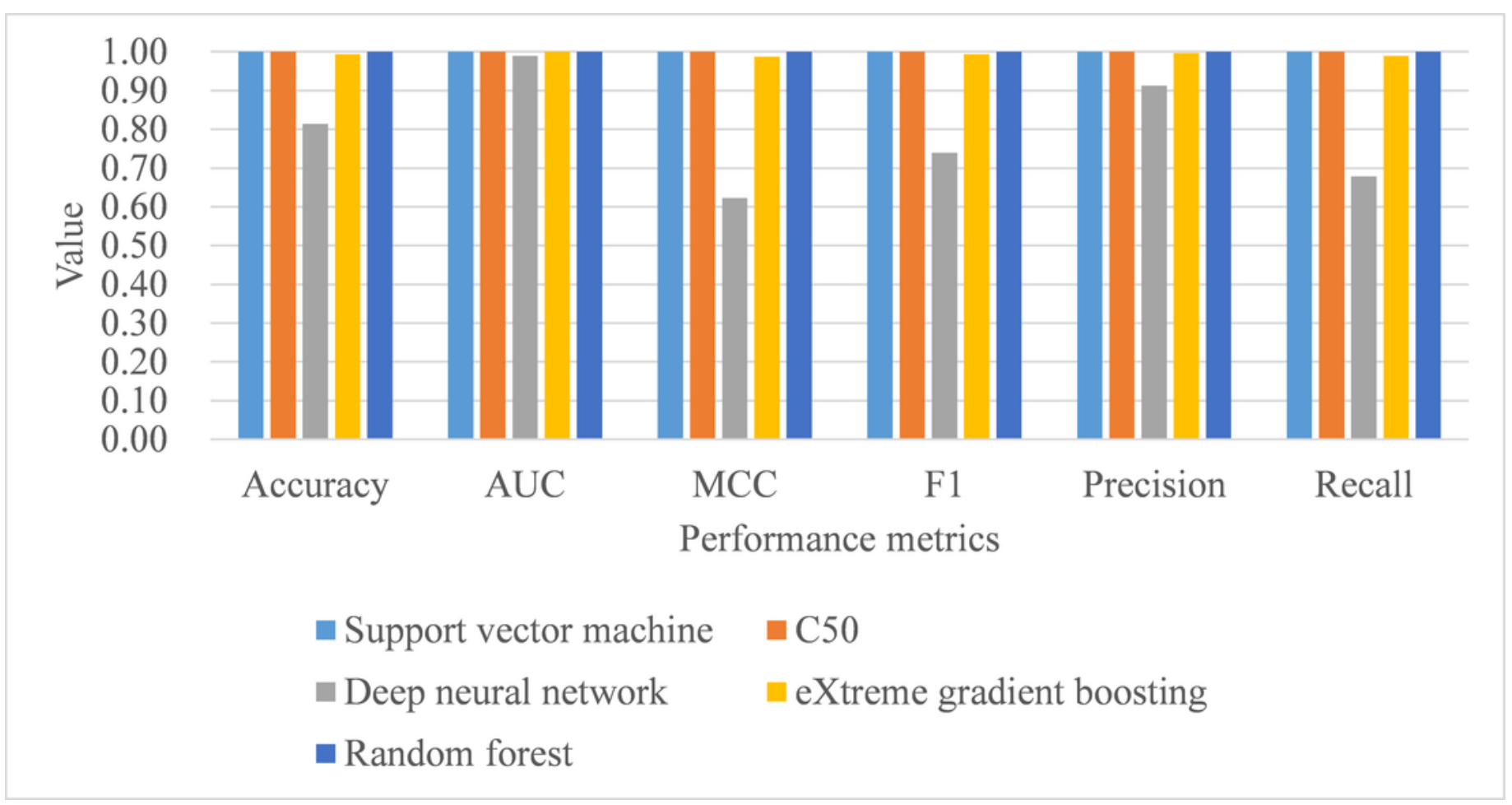


Figure 7

Fig. 7 Importance of features

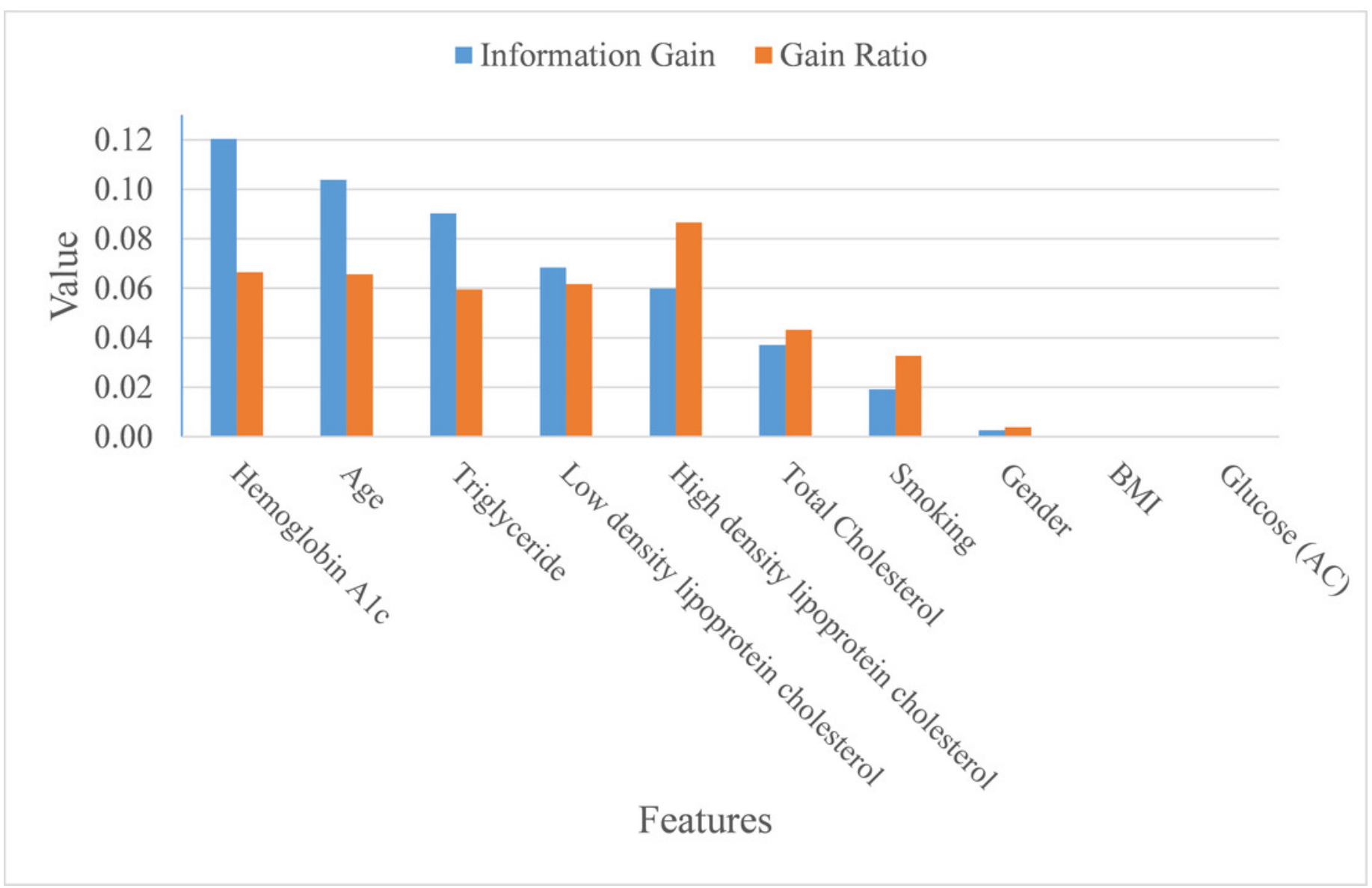




\section{Table 1 (on next page)}

Table 1. Type 2 diabetes mellitus diagnosis related studies: Adopted machine learning algorithms 
1 Table 1. Type 2 diabetes mellitus diagnosis related studies: Adopted machine learning algorithms

\begin{tabular}{|c|c|c|c|c|c|c|c|}
\hline Study & Instance-based & Decision trees & $\begin{array}{l}\text { Neural } \\
\text { network }\end{array}$ & Ensemble & Bayesian & Statistical model & Others \\
\hline Pei et al. (2019) & $\begin{array}{l}\text { Support vector } \\
\text { machine }\end{array}$ & J48* & & Adaboostm 1 & $\begin{array}{l}\text { Naïve } \\
\text { Bayes, } \\
\text { Bayes net }\end{array}$ & & \\
\hline Wu et al. (2018) & & & & & & $\begin{array}{l}\text { Logistic } \\
\text { regression }\end{array}$ & K-means \\
\hline $\begin{array}{l}\text { Talaei-Khoei \& } \\
\text { Wilson (2018) }\end{array}$ & $\begin{array}{l}\text { Support vector } \\
\text { machine* }\end{array}$ & $\begin{array}{l}\text { Decision } \\
\text { trees }\end{array}$ & $\begin{array}{l}\text { Neural } \\
\text { network* }\end{array}$ & & & $\begin{array}{l}\text { Logistic } \\
\text { regression* }\end{array}$ & Clustering \\
\hline $\begin{array}{l}\text { Upadhyaya et al. } \\
\text { (2017) }\end{array}$ & & & & & & & $\begin{array}{l}\text { First-order logic } \\
\text { rules }\end{array}$ \\
\hline $\begin{array}{l}\text { Nilashi et al. } \\
(2017)\end{array}$ & $\begin{array}{l}\text { Self-organizing } \\
\text { map, support } \\
\text { vector machine }\end{array}$ & & $\begin{array}{l}\text { Neural } \\
\text { network* }\end{array}$ & & & & $\begin{array}{l}\text { Principal } \\
\text { component analysis }\end{array}$ \\
\hline $\begin{array}{l}\text { Maniruzzaman et } \\
\text { al. (2017) }\end{array}$ & & & & & $\begin{array}{l}\text { Naïve } \\
\text { Bayes }\end{array}$ & $\begin{array}{l}\text { Linear } \\
\text { discriminant } \\
\text { analysis, } \\
\text { Quadratic } \\
\text { discriminant } \\
\text { analysis }\end{array}$ & $\begin{array}{l}\text { Gaussian process } \\
\text { classification* }\end{array}$ \\
\hline $\begin{array}{l}\text { Kagawa et al. } \\
(2017)\end{array}$ & $\begin{array}{l}\text { Support vector } \\
\text { machine }\end{array}$ & & & & & & $\begin{array}{l}\text { Rule-based*, } \\
\text { Modified PheKB }\end{array}$ \\
\hline
\end{tabular}

2 Note: * denotes the best performed algorithm. 
3 Table 1. Type 2 diabetes mellitus diagnosis related studies: Adopted machine learning algorithms (cont.)

\begin{tabular}{|c|c|c|c|c|c|c|c|}
\hline Study & Instance-based & Decision trees & $\begin{array}{l}\text { Neural } \\
\text { network }\end{array}$ & Ensemble & Bayesian & Statistical model & Others \\
\hline $\begin{array}{l}\text { Alghamdi et al. } \\
\text { (2017) }\end{array}$ & & $\begin{array}{l}\mathrm{J} 48 \text {, Decision } \\
\text { tree, Logistic } \\
\text { model tree }\end{array}$ & & $\begin{array}{l}\text { Random } \\
\text { forest }\end{array}$ & $\begin{array}{l}\text { Naïve } \\
\text { Bayes }\end{array}$ & $\begin{array}{l}\text { Logistic } \\
\text { regression* }\end{array}$ & \\
\hline $\begin{array}{l}\text { Esteban et al. } \\
(2017)\end{array}$ & $\begin{array}{l}\text { Support vector } \\
\text { machine, KNN }\end{array}$ & C5.0 & $\begin{array}{l}\text { Neural } \\
\text { networks* }\end{array}$ & $\begin{array}{l}\text { Random } \\
\text { forest, } \\
\text { Gradient } \\
\text { boosting } \\
\text { machine, } \\
\text { Extreme } \\
\text { gradient } \\
\text { boosting }\end{array}$ & $\begin{array}{l}\text { Bayesian } \\
\text { model }\end{array}$ & $\begin{array}{l}\text { Linear model, } \\
\text { Discriminant } \\
\text { analysis, Partial } \\
\text { least squares, } \\
\text { Multinomial } \\
\text { logistic regression }\end{array}$ & $\begin{array}{l}\text { Rule-based, Elastic } \\
\text { net, Nearest } \\
\text { shrunken centroid }\end{array}$ \\
\hline $\begin{array}{l}\text { Anderson et al. } \\
(2015)\end{array}$ & & & & & $\begin{array}{l}\text { Bayesian } \\
\text { inference }\end{array}$ & & \\
\hline
\end{tabular}

4 Note: * denotes the best performed algorithm. 


\section{Table 2 (on next page)}

Table 2. Type 2 diabetes mellitus diagnosis-related studies: Included features for machine learning 
1 Table 2. Type 2 diabetes mellitus diagnosis-related studies: Included features for machine learning

\begin{tabular}{|c|c|c|c|c|c|c|}
\hline Study & Demographic data & Laboratory test results & Vital signs & Life style & History & Others \\
\hline Pei et al. (2019) & Age, gender, BMI & & & $\begin{array}{l}\text { Physical activity, } \\
\text { work stress, salty } \\
\text { food preference }\end{array}$ & $\begin{array}{l}\text { History of cardiovascular } \\
\text { disease or stroke, family } \\
\text { history of diabetes, } \\
\text { hypertension }\end{array}$ & \\
\hline Wu et al. (2018) & Age, BMI & $\begin{array}{l}\text { 2-h plasma glucose, 2-h } \\
\text { serum insulin, diabetes } \\
\text { pedigree function }\end{array}$ & $\begin{array}{l}\text { Diastolic } \\
\text { blood } \\
\text { pressure }\end{array}$ & & Number of times pregnant & $\begin{array}{l}\text { Triceps skin } \\
\text { fold thickness }\end{array}$ \\
\hline $\begin{array}{l}\text { Talaei-Khoei \& } \\
\text { Wilson (2018) }\end{array}$ & Age, sex, BMI & $\begin{array}{l}\text { High density lipoprotein } \\
\text { cholesterol, triglycerides, } \\
\text { fast plasma glucose, } \\
\text { Hemoglobin A1c }\end{array}$ & $\begin{array}{l}\text { Systolic } \\
\text { blood } \\
\text { pressure }\end{array}$ & $\begin{array}{l}\text { Shortness of } \\
\text { breath, frequent } \\
\text { urination at } \\
\text { night, excessive } \\
\text { thirst }\end{array}$ & $\begin{array}{l}\text { History of high blood } \\
\text { glucose, parental history } \\
\text { of diabetes }\end{array}$ & $\begin{array}{l}\text { Waist/hip } \\
\text { ratio, waist } \\
\text { circumference }\end{array}$ \\
\hline $\begin{array}{l}\text { Upadhyaya et al. } \\
\text { (2017) }\end{array}$ & & Hemoglobin A1c & & & $\begin{array}{l}\text { A prescription for } \\
\text { metformin, DM-related } \\
\text { medication }\end{array}$ & $\begin{array}{l}\text { ICD-9-CM } \\
\text { code }\end{array}$ \\
\hline $\begin{array}{l}\text { Nilashi et al. } \\
\text { (2017) }\end{array}$ & Age, sex, BMI & $\begin{array}{l}\text { High density lipoprotein } \\
\text { cholesterol, triglycerides, } \\
\text { fast plasma glucose, } \\
\text { Hemoglobin A1c }\end{array}$ & $\begin{array}{l}\text { Systolic } \\
\text { blood } \\
\text { pressure }\end{array}$ & $\begin{array}{l}\text { Shortness of } \\
\text { breath, frequent } \\
\text { urination at } \\
\text { night, excessive } \\
\text { thirst }\end{array}$ & $\begin{array}{l}\text { History of high blood } \\
\text { glucose, parental history } \\
\text { of diabetes }\end{array}$ & $\begin{array}{l}\text { Waist/hip } \\
\text { ratio, waist } \\
\text { circumference }\end{array}$ \\
\hline
\end{tabular}


3 Table 2. Type 2 diabetes mellitus diagnosis related studies: Included features for machine learning (cont.)

\begin{tabular}{|c|c|c|c|c|c|c|}
\hline Study & Demographic data & Laboratory test results & Vital signs & Life style & History & Others \\
\hline \multirow{5}{*}{$\begin{array}{l}\text { Maniruzzaman et } \\
\text { al. (2017) }\end{array}$} & \multirow[t]{4}{*}{ Age, sex, BMI } & High density lipoprotein & \multirow{4}{*}{$\begin{array}{l}\text { Systolic } \\
\text { blood } \\
\text { pressure }\end{array}$} & Shortness of & \multirow{3}{*}{$\begin{array}{l}\text { History of high blood } \\
\text { glucose, parental history } \\
\text { of diabetes }\end{array}$} & \multirow{4}{*}{$\begin{array}{l}\text { Waist/hip } \\
\text { ratio, waist } \\
\text { circumference }\end{array}$} \\
\hline & & cholesterol, triglycerides, fast & & breath, frequent & & \\
\hline & & plasma glucose, Hemoglobin & & urination at & & \\
\hline & & Alc & & night, excessive & & \\
\hline & & & & thirst & & \\
\hline
\end{tabular}

Kagawa et al.

(2017)

Random glucose, glycol-

albumin, HbA1c, GAD

antibody, IA2 antibody, C-

peptide

Alghamdi et al. Age, black, Metabolic equivalent

(2017) obesity

Esteban et al.

(2017)

Fasting glycemia, $\mathrm{HbA1c}$
T2DM medication

ICD-10 code, I251-insulin

biding ratio

Resting heart Sedentary
rate, resting lifestyle
systolic blood
pressure,
resting
diastolic blood
pressure

Diabetes Mellitus related Diabetes prescriptions filled Mellitus 
5 Table 2. Type 2 diabetes mellitus diagnosis related studies: Included features for machine learning (cont.)

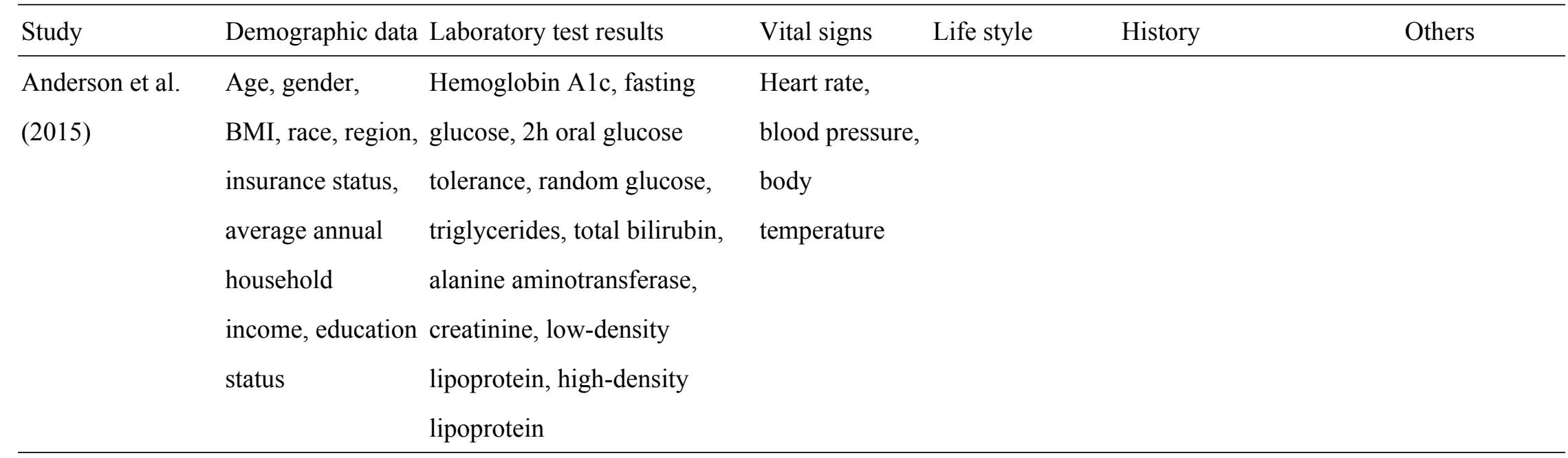




\section{Table 3 (on next page)}

Table 3. Type 2 diabetes mellitus diagnosis-related studies: Samples and classification type 
1 Table 3. Type 2 diabetes mellitus diagnosis-related studies: Samples and classification type

\begin{tabular}{|c|c|c|c|c|}
\hline Study & Country & Sample size & Classification type & Results \\
\hline Pei et al. (2019) & China & 4,205 & Binary & $\begin{array}{l}\mathrm{J} 48 \text { has the best performance }(\text { accuracy }=0.9503, \text { precision }= \\
0.950, \text { recall }=0.950, \text { F-measure }=0.948, \text { and AUC }=0.964)\end{array}$ \\
\hline Wu et al. (2018) & USA & 768 & Binary & $\begin{array}{l}\text { The proposed model attained a } 3.04 \% \text { higher prediction } \\
\text { accuracy than those of other studies }\end{array}$ \\
\hline Talaei-Khoei \& & Australia & 10,911 & Binary & $\begin{array}{l}\text { The performance of different learners depends on both period } \\
\text { and purpose of prediction }\end{array}$ \\
\hline \multicolumn{5}{|l|}{ Wilson (2018) } \\
\hline $\begin{array}{l}\text { Upadhyaya et al. } \\
(2017)\end{array}$ & USA & 4,208 & Binary & $\begin{array}{l}\text { The proposed algorithm performed well with a } 99.70 \% \\
\text { sensitivity and a } 99.97 \% \text { specificity }\end{array}$ \\
\hline Nilashi et al. (2017) & USA & 768 & Binary & $\begin{array}{l}\text { The proposed method remarkably improves the accuracy of } \\
\text { prediction in relation to prior methods }\end{array}$ \\
\hline $\begin{array}{l}\text { Maniruzzaman et al. } \\
(2017)\end{array}$ & USA & 768 & Binary & $\begin{array}{l}\text { The performance of Gaussian process classification are better } \\
\text { than other methods with accuracy }=81.97 \% \text {, sensitivity }= \\
91.79 \% \text {, positive predictive value }=84.91 \% \text {, and negative } \\
\text { predictive value }=62.50 \%\end{array}$ \\
\hline Kagawa et al. (2017) & Japan & 104,522 & Binary & $\begin{array}{l}\text { The proposed phenotyping algorithms show better } \\
\text { performance than baseline algorithms }\end{array}$ \\
\hline $\begin{array}{l}\text { Alghamdi et al. } \\
(2017)\end{array}$ & USA & 32,555 & Binary & $\begin{array}{l}\text { The proposed ensemble approach achieved high accuracy of } \\
\text { prediction }(A U C=0.920)\end{array}$ \\
\hline Esteban et al. (2017) & Argentina & 2,463 & Multi-class & $\begin{array}{l}\text { The stacked generalization strategy and feed-forward neural } \\
\text { network performed the best with validation set. }\end{array}$ \\
\hline $\begin{array}{l}\text { Anderson et al. } \\
(2015)\end{array}$ & USA & 24,331 & Binary & $\begin{array}{l}\text { The proposed ensemble model accurately predicted } \\
\text { progression to T2DM }(\text { AUC }=0.76) \text {, and was validated out of } \\
\text { sample }(\text { AUC }=0.78)\end{array}$ \\
\hline
\end{tabular}


Table 4 (on next page)

Table 4. Operational definition of features 
1 Table 4. Operational definition of features

\begin{tabular}{|c|c|c|c|c|}
\hline \multicolumn{2}{|c|}{ Features/Target class } & \multirow{2}{*}{$\frac{\text { Measurement }}{\text { Discrete }}$} & \multirow{2}{*}{$\begin{array}{l}\text { Definition } \\
\text { The probability of four kinds of T2DM diagnosis: E1121, } \\
\text { E1143, and E1165 }\end{array}$} & \multirow{2}{*}{$\begin{array}{l}\text { References } \\
\text { NA }\end{array}$} \\
\hline $\begin{array}{l}\text { Target } \\
\text { class }\end{array}$ & $\begin{array}{l}\text { Diagnosis of } \\
\text { T2DM }\end{array}$ & & & \\
\hline \multirow[t]{6}{*}{ Features } & Gender & Discrete & Gender of the patients, Male or Female. & $\begin{array}{l}\text { Anderson et al. (2015); } \\
\text { Pei et al. (2019); Wu et al. } \\
\text { (2018) }\end{array}$ \\
\hline & Age & Continuous & Age (in years) during out-patient services & $\begin{array}{l}\text { Anderson et al. (2015); } \\
\text { Pei et al. (2019); Talaei- } \\
\text { Khoei \& Wilson (2018) }\end{array}$ \\
\hline & $\begin{array}{l}\text { Smoking } \\
\text { status }\end{array}$ & Discrete & Yes, quit, or no & \\
\hline & BMI & Continuous & Body mass index & $\begin{array}{l}\text { Anderson et al. (2015); } \\
\text { Pei et al. (2019); Wu et } \\
\text { al. (2018) }\end{array}$ \\
\hline & $\begin{array}{l}\text { Total } \\
\text { Cholesterol }\end{array}$ & Continuous & The level of total cholesterol during out-patient services & \\
\hline & Triglyceride & Continuous & The level of triglyceride during out-patient services & $\begin{array}{l}\text { Anderson et al. (2015); } \\
\text { Talaei-Khoei \& Wilson } \\
\text { (2018) }\end{array}$ \\
\hline
\end{tabular}


3 Table 4. Operational definition of features (cont.)

\begin{tabular}{|c|c|c|c|}
\hline Features/Target class & Measurement & Definition & References \\
\hline Glucose (AC) & Continuous & The level of glucose (AC) during out-patient services & Anderson et al. (2015) \\
\hline Hemoglobin & Continuous & The level of Hemoglobin A1c during out-patient services & Anderson et al. (2015); \\
\hline \multirow[t]{2}{*}{ Alc } & & & Kagawa et al. (2017); \\
\hline & & & $\begin{array}{l}\text { Talaei-Khoei \& Wilson } \\
\text { (2018) }\end{array}$ \\
\hline High density & Continuous & The level of high-density lipoprotein cholesterol during out- & Anderson et al. (2015); \\
\hline lipoprotein & & patient services & Talaei-Khoei \& Wilson \\
\hline cholesterol & & & $(2018)$ \\
\hline Low density & Continuous & The level of low-density lipoprotein cholesterol during out- & Anderson et al. (2015) \\
\hline lipoprotein & & patient services & \\
\hline cholesterol & & & \\
\hline
\end{tabular}

4 


\section{Table 5 (on next page)}

Table 5. R packages used and the optimal model parameters given 
1 Table 5. R packages used and the optimal model parameters given

\begin{tabular}{|c|c|c|c|}
\hline Method & Parameters & $\begin{array}{l}\text { Best parameter } \\
\text { setting }\end{array}$ & R packages \\
\hline Support vector & sigma & 0.664667494 & kernlab 0.9-29 \\
\hline machine & $\mathrm{C}$ & 11.07262251 & \\
\hline \multirow[t]{2}{*}{ C5.0 } & winnow & FALSE & C50 0.1.3 \\
\hline & trials & 43 & \\
\hline Deep neural & hidden & 200 & h2o 3.30.0.1 \\
\hline \multirow[t]{2}{*}{ network } & input_dropout_ratio & 0 & \\
\hline & activation & Maxout & \\
\hline eXtreme gradient & nrounds & 154 & xgboost 1.0.0.2 \\
\hline \multirow[t]{6}{*}{ boosting } & max_depth & 10 & \\
\hline & eta & 0.745922343 & \\
\hline & gamma & 3.194824195 & \\
\hline & colsample_bytree & 0.945590117 & \\
\hline & min_child_weight & 3.35705624 & \\
\hline & subsample & 0.802348509 & \\
\hline Random Forest & mtry & 2 & randomForest 4.6-14 \\
\hline
\end{tabular}

2 
Table 6(on next page)

Table 6. Confusion matrix 
1 Table 6. Confusion matrix

\begin{tabular}{llll}
\hline & & Predicted class & \\
& & Positive & Negative \\
\hline Actual class & Positive & True positive (TP) & False negative (FN) \\
& Negative & False positive (FP) & True negative (TN) \\
\hline
\end{tabular}

2

3 
Table 7 (on next page)

Table 7. Formulae for performance metrics 
1 Table 7. Formulae for performance metrics

\begin{tabular}{|c|c|}
\hline Metric & Formula \\
\hline \multirow[t]{3}{*}{ Average accuracy } & $\mathrm{TP}_{i}+\mathrm{TN}_{i}$ \\
\hline & $Z_{i=1} \overline{\mathrm{TP}_{i}+\mathrm{FN}_{i}+\mathrm{FP}_{i}+\mathrm{TN}_{i}}$ \\
\hline & $\bar{l}$ \\
\hline \multirow{2}{*}{$\begin{array}{l}\text { Matthew correlation } \\
\text { coefficient }\end{array}$} & $(\mathrm{TP} * \mathrm{TN}+\mathrm{FP} * \mathrm{FN})$ \\
\hline & $\overline{\operatorname{Sqrt}((\mathrm{TP}+\mathrm{FP}) *(\mathrm{TP}+\mathrm{FN}) *(\mathrm{TN}+\mathrm{FP}) *(\mathrm{TN}+\mathrm{FN}))}$ \\
\hline \multirow[t]{3}{*}{ Precision $_{\mathbf{M}}$} & $\mathrm{TP}_{i}$ \\
\hline & $L_{i=1} \overline{\left(\mathrm{TP}_{i}+\mathrm{FP}_{i}\right)}$ \\
\hline & $\bar{l}$ \\
\hline \multirow[t]{3}{*}{ Recall $_{\mathbf{M}}$} & $\Gamma^{l} \quad \mathrm{TP}_{i}$ \\
\hline & $\square_{i=1}\left(\mathrm{TP}_{i}+\mathrm{FN}_{i}\right)$ \\
\hline & $\bar{l}$ \\
\hline \multirow[t]{2}{*}{ F1 score $_{\mathbf{M}}$} & $2 * \operatorname{Precision}_{\mathrm{M}} * \operatorname{Recall}_{\mathrm{M}}$ \\
\hline & Precision $_{M}+$ Recall $_{M}$ \\
\hline
\end{tabular}

2 Note: 1 denotes class levels, $\mathrm{M}$ denotes macro-averaging metrics, TP means true positive, FP 3 denotes false positive, FN means false negative, and TN denotes true negative.

4 


\section{Table 8 (on next page)}

Table 8. Data summary results 
1 Table 8. Data summary results

\begin{tabular}{lll}
\hline Feature & Range & Summary statistics \\
\hline Gender & Male / Female & Male: 86, Female: 63 \\
Age & $21 \sim 91$ & $\mathrm{M}=61.27, \mathrm{SD}=13.70$ \\
Smoking status & No / Quit / Yes & No $=123$, Quit $=10, \mathrm{Yes}=16$ \\
BMI & $15.49 \sim 44.05$ & $\mathrm{M}=26.63, \mathrm{SD}=4.77$ \\
Total Cholesterol & $77 \sim 311$ & $\mathrm{M}=151.98, \mathrm{SD}=34.38$ \\
Triglyceride & $37 \sim 546$ & $\mathrm{M}=136.64, \mathrm{SD}=93.30$ \\
Glucose (AC) & $68 \sim 346$ & $\mathrm{M}=146.58, \mathrm{SD}=51.72$ \\
Hemoglobin A1c & $5.1 \sim 11.6$ & $\mathrm{M}=7.46, \mathrm{SD}=1.21$ \\
High density lipoprotein cholesterol & $16 \sim 98$ & $\mathrm{M}=47.44, \mathrm{SD}=14.87$ \\
Low density lipoprotein cholesterol & $29 \sim 152$ & $\mathrm{M}=71.42, \mathrm{SD}=25.66$ \\
\hline
\end{tabular}

2 Note: $\mathrm{M}$ denotes mean and SD means standard deviation. 
Table 9 (on next page)

Table 9. Model performance: 10-fold cross-validation 
Table 9. Model performance: 10-fold cross-validation

\begin{tabular}{|c|c|c|c|c|c|c|c|c|c|}
\hline \multirow{2}{*}{ Sample } & \multirow{2}{*}{ Learner } & \multirow{2}{*}{ Accuracy(SD) } & \multirow{2}{*}{ AUC(SD) } & \multirow{2}{*}{$\mathrm{MCC}(\mathrm{SD})$} & \multicolumn{3}{|c|}{ Macro } & \multirow{2}{*}{$\begin{array}{l}\text { Process } \\
\text { time }\end{array}$} & \multirow{2}{*}{$\begin{array}{l}\text { Stuart-Maxwell } \\
\text { test }\end{array}$} \\
\hline & & & & & $\mathrm{F} 1(\mathrm{SD})$ & Precision(SD) & Recall(SD) & & \\
\hline \multirow[t]{5}{*}{ Train } & SVM & $0.998(0.006)$ & $1.000(0.000)$ & $0.995(0.011)$ & $0.994(0.012)$ & $0.997(0.008)$ & $0.991(0.015)$ & 2.22 & \\
\hline & C5.0 & $0.984(0.015)$ & $0.999(0.001)$ & $0.969(0.031)$ & $0.981(0.020)$ & $0.987(0.015)$ & $0.975(0.026)$ & 6.74 & \\
\hline & $\mathrm{DNN}$ & $0.947(0.019)$ & $0.985(0.016)$ & $0.896(0.033)$ & $0.935(0.027)$ & $0.956(0.031)$ & $0.922(0.028)$ & 13.56 & \\
\hline & XGB & $0.943(0.021)$ & $0.992(0.008)$ & $0.885(0.044)$ & $0.918(0.050)$ & $0.946(0.036)$ & $0.894(0.058)$ & 7.86 & \\
\hline & $\mathrm{RF}$ & $0.986(0.010)$ & $1.000(0.000)$ & $0.972(0.017)$ & $0.985(0.011)$ & $0.992(0.006)$ & $0.978(0.016)$ & 4.59 & \\
\hline \multirow[t]{9}{*}{ Test } & SVM & 1.000 & 1.000 & 1.000 & 1.000 & 1.000 & 1.000 & & \\
\hline & $\mathrm{C} 5.0$ & 1.000 & 1.000 & 1.000 & 1.000 & 1.000 & 1.000 & & \\
\hline & $\mathrm{DNN}$ & 0.855 & 0.985 & 0.730 & 0.678 & 0.876 & 0.684 & & $\chi^{2}(3)=$ \\
\hline & & & & & & & & & $253.20, p<$ \\
\hline & & & & & & & & & 0.001 \\
\hline & XGB & 0.989 & 1.000 & 0.979 & 0.985 & 0.992 & 0.978 & & $\chi^{2}(2)=$ \\
\hline & & & & & & & & & $13.00, p=$ \\
\hline & & & & & & & & & 0.002 \\
\hline & $\mathrm{RF}$ & 1.000 & 1.000 & 1.000 & 1.000 & 1.000 & 1.000 & & \\
\hline
\end{tabular}

2 Note: AUC: area under receiver operating characteristic, SD: standard deviation, MCC: Matthew correlation coefficient, SVM: support

3 vector machine, DNN: deep neural network, XGB: eXtreme gradient boosting, RF: random forest, the second is used to measure process 4 time. 
Table $\mathbf{1 0}$ (on next page)

Table 10. Model performance: leave-one-subject-out cross-validation 
Table 10. Model performance: leave-one-subject-out cross-validation

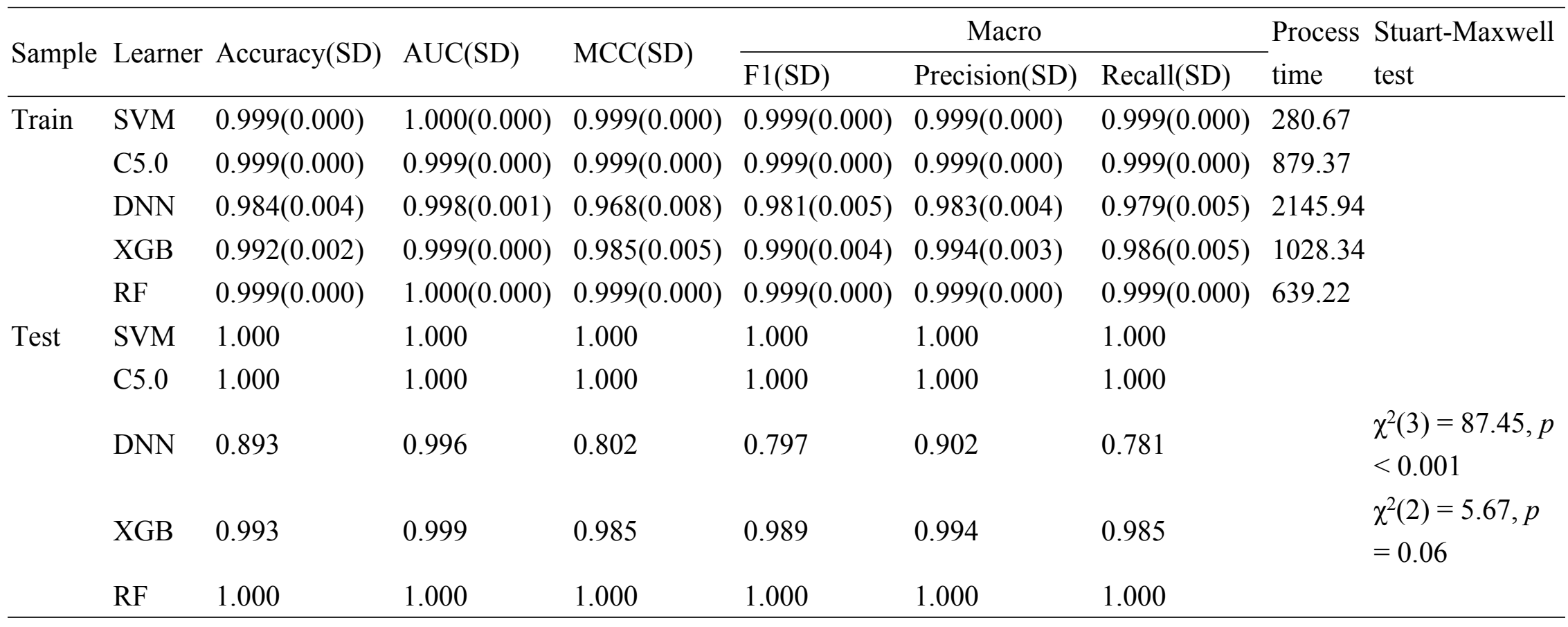

2 Note: AUC: area under receiver operating characteristic, SD: standard deviation, MCC: Matthew correlation coefficient, SVM: support

3 vector machine, DNN: deep neural network, XGB: eXtreme gradient boosting, RF: random forest, the second is used to measure process 4 time. 


\section{Table 11 (on next page)}

Table 11. Model performance: holdout cross-validation 
1 Table 11. Model performance: holdout cross-validation

\begin{tabular}{|c|c|c|c|c|c|c|c|c|}
\hline \multirow{2}{*}{ Sample } & \multirow{2}{*}{ Method } & \multirow{2}{*}{ Accuracy } & \multirow{2}{*}{ AUC } & \multirow{2}{*}{$\mathrm{MCC}$} & \multicolumn{3}{|c|}{ Macro } & \multirow{2}{*}{-Process time $\begin{array}{l}\text { Stuart- } \\
\text { Maxwell test }\end{array}$} \\
\hline & & & & & F1 & Precision & Recall & \\
\hline \multirow[t]{5}{*}{ Train } & SVM & 1.000 & 1.000 & 1.000 & 1.000 & 1.000 & 1.000 & 0.23 \\
\hline & C5.0 & 0.950 & 0.996 & 0.903 & 0.933 & 0.948 & 0.920 & 0.59 \\
\hline & DNN & 0.970 & 0.997 & 0.940 & 0.954 & 0.970 & 0.939 & 1.59 \\
\hline & XGB & 0.886 & 0.974 & 0.775 & 0.809 & 0.869 & 0.770 & 0.75 \\
\hline & $\mathrm{RF}$ & 0.978 & 1.000 & 0.957 & 0.980 & 0.989 & 0.972 & 0.39 \\
\hline \multirow[t]{5}{*}{ Test } & SVM & 1.000 & 1.000 & 1.000 & 1.000 & 1.000 & 1.000 & \\
\hline & $\mathrm{C} 5.0$ & 1.000 & 1.000 & 1.000 & 1.000 & 1.000 & 1.000 & \\
\hline & $\mathrm{DNN}$ & 0.814 & 0.989 & 0.623 & 0.739 & 0.913 & 0.676 & $\chi^{2}(3)=205.04, p<0.001$ \\
\hline & XGB & 0.993 & 1.000 & 0.987 & 0.993 & 0.996 & 0.989 & $\chi^{2}(2)=8.00, p=0.018$ \\
\hline & $\mathrm{RF}$ & 1.000 & 1.000 & 1.000 & 1.000 & 1.000 & 1.000 & \\
\hline
\end{tabular}

2 Note: AUC: area under receiver operating characteristic, SD: standard deviation, MCC: Matthew correlation coefficient, SVM: support

3 vector machine, DNN: deep neural network, XGB: eXtreme gradient boosting, RF: random forest, the second is used to measure process

4 time 


\section{Table 12 (on next page)}

Table 12. Comparison of our study with state-of-the-art works 
1 Table 12. Comparison of our study with state-of-the-art works

\begin{tabular}{llllllll}
\hline Algorithms & Study & Accuracy & AUC & MCC & Precision Recall & F1 score \\
\hline $\begin{array}{l}\text { Support } \\
\text { vector }\end{array}$ & This study & 1 & 1 & 1 & 1 & 1 & 1 \\
machine & Pei et al. (2019) & 0.908 & 0.763 & NA & 0.903 & 0.908 & 0.905 \\
& $\begin{array}{l}\text { Talaei-Khoei \& Wilson } \\
\text { (2018) }\end{array}$ & NA & 0.831 & 0.922 & NA & 0.683 & NA \\
& Kagawa et al. (2017) & NA & NA & NA & 0.8 & 0.909 & NA \\
\hline Neural & This study & 0.788 & 0.986 & 0.566 & 0.910 & 0.620 & 0.684 \\
network & Talaei-Khoei \& Wilson & NA & 0.663 & 0.007 & NA & 0.41 & NA \\
& (2018) & & & & & & \\
& Nilashi et al. (2017) & 0.923 & NA & NA & NA & NA & NA \\
& Esteban et al. (2017) & NA & NA & NA & 0.930 & 0.960 & 0.940 \\
\hline $\begin{array}{l}\text { Random } \\
\text { forest }\end{array}$ & This study & 1 & 1 & 1 & 1 & 1 & 1 \\
\hline
\end{tabular}

2 Note: AUC: area under receiver operating characteristic, MCC: Matthew correlation coefficient,

3 NA: not available. 